\title{
Investigation of the Ultrafast Response and Saturable Absorption of Voltage-Controlled Graphene
}

\author{
I. Baylam ${ }^{1}$, M.N. Cizmeciyan ${ }^{1}$, N. Kakenov ${ }^{2}$, C. Kocabas ${ }^{2}$, and A. Sennaroglu, ${ }^{1,3, *}$ \\ ${ }^{I}$ Koç University Surface Science and Technology Center (KUYTAM), Rumelifeneri, Istanbul 34450, Turkey \\ ${ }^{2}$ Department of Physics, Bilkent University, Ankara 06800, Turkey \\ ${ }^{3}$ Laser Research Laboratory, Departments of Physics and Electrical-Electronics Engineering, Koç University, Istanbul 34450, Turkey \\ Corresponding author: asennar@ku.edu.tr
}

\begin{abstract}
Ultrafast pump-probe measurements show that at a bias voltage of $1 \mathrm{~V}$, voltage reconfigurable graphene supercapacitors can operate as fast saturable absorbers with adjustable insertion loss over an ultrabroad spectral range from 630 to $1100 \mathrm{~nm}$. $\odot 2018$ The Author(s)

OCIS codes: (190.7110) Ultrafast nonlinear optics; (320.7150) Ultrafast spectroscopy; (160.4330) Nonlinear optical materials.
\end{abstract}

\section{Introduction}

Recently, nonlinear optical properties of 2D layered materials have been widely investigated for various applications [1]. In the particular case of graphene, graphene-based supercapacitor structures were shown to operate as fast saturable absorbers for femtosecond pulse generation [2,3]. Furthermore, the insertion loss of this class of saturable absorbers could be adjusted with only a few volts of bias in the $0-2 \mathrm{~V}$ range. Since the applied bias and the resulting shift in the Fermi level may also affect the absorption saturation behavior of these devices, it is important to experimentally determine the spectral window over which they exhibit fast saturable absorption at different levels of bias. In this study, we investigated the ultrafast nonlinear response of a voltage-controlled graphene-gold saturable absorber (VCG-gold-SA) by using femtosecond pump probe spectroscopy. Visible and near-infrared continuum probe pulses covering the wavelength range of 500-1600 nm were used. In the experiments, we observed both saturable absorption and multiphoton absorption regimes as the applied bias voltage was varied. Bias-dependent saturation fluence, modulation depth and decay times of the VCG-gold-SA were further measured. Measurements indicated that with increasing bias voltage, the saturable absorption bandwidth decreased but remained sufficiently wide to generate femtosecond pulses. In particular, at the bias voltage of $1 \mathrm{~V}$, the VCG-gold-SA could be operated as a fast saturable absorber with reduced insertion loss over the wavelength range of 630-1100 $\mathrm{nm}$.

\section{Experimental setup and results}

In the experiments, a femtosecond transient absorption spectrometer (Ultrafast Systems, Helios) operating around visible and near-infrared wavelengths was used to investigate the voltage-dependent nonlinear and ultrafast absorption characteristics of the VCG-gold-SA. The pump pulses were obtained from a tunable optical parametric amplifer (Spectra-Physics, TOPAS Prime) which was in turn pumped with a $1-\mathrm{kHz}, 800-\mathrm{nm} \mathrm{Ti}^{3+}$ :sapphire regenerative amplifier (Spectra-Physics, Spitfire Ace, pulsewidth=134 fs). Visible $(500-800 \mathrm{~nm}$ ) and near-infrared (800-1600 nm) white-light continuum pulses were used to measure the nonlinear absorption spectrum $\Delta \mathrm{A}$ ( $\triangle \mathrm{A}=$ pumped absorption-unpumped absorption) as a function of pump energy and pump-probe delay at different levels of applied bias voltage. We observed both saturable absorption $(\Delta \mathrm{A}<0)$ and multiphoton absorption $(\Delta \mathrm{A}>0)$ regimes [4] for the VCG-gold-SA at different applied voltages. To determine the voltage-dependent operation bandwidth in which the VCG-gold acted as a saturable absorber, we used a pump wavelength of $470 \mathrm{~nm}$ and 700 $\mathrm{nm}$, respectively, for the visible and near-infrared continuum pulses. Bias-dependent $\Delta \mathrm{A}$ spectra are displayed in Figs. 1(a) and 1(b). Based on the data in Figs. 1(a) and 1(b), we see that the saturable absorption bandwidth (ie the wavelength range where $\Delta \mathrm{A}$ remains negative) of the VCG-gold-SA decreases with increasing bias voltage. Combining the data of Figs. 1(a) and 1(b), the saturable absorption band extends from $630 \mathrm{~nm}$ to $1100 \mathrm{~nm}$ at the bias voltage of $1 \mathrm{~V}$. In addition, voltage-dependent saturation fluence and modulation depth of the VCG-gold-SA were measured within the saturable absorption regime $(\Delta \mathrm{A}<0)$ at the pump wavelengths of $650 \mathrm{~nm}$ and $1000 \mathrm{~nm}$. At zero bias, the saturation fluence and single-pass modulation depth for the $650-\mathrm{nm}$ pump were determined to be 682 $\mu \mathrm{J} / \mathrm{cm}^{2}$ and $0.52 \%$, respectively. In agreement with previous results [4], the saturation fluence $\left(67.5 \mu \mathrm{J} / \mathrm{cm}^{2}\right)$ and the single-pass modulation depth $(0.27 \%)$ came to be lower for the 1000-nm pump. Figure 1(c) shows that the singlepass modulation depth of the VCG-gold-SA monotonically decreased from $0.52 \%(0.27 \%)$ to $0.24 \%(0.16 \%)$ as the applied bias was increased from 0 to $1.6 \mathrm{~V}(0.8 \mathrm{~V})$ for the $650-\mathrm{nm}(1000-\mathrm{nm})$ pump pulse. The zero-bias saturation fluence at the 650-nm (1000-nm) pump wavelength was around $682 \mu \mathrm{J} / \mathrm{cm}^{2}\left(67.5 \mu \mathrm{J} / \mathrm{cm}^{2}\right)$ and increased to 951 $\mu \mathrm{J} / \mathrm{cm}^{2}\left(168 \mu \mathrm{J} / \mathrm{cm}^{2}\right)$ for the applied voltage of $1.6 \mathrm{~V}(0.8 \mathrm{~V})$ [see Fig. 1(c)]. 

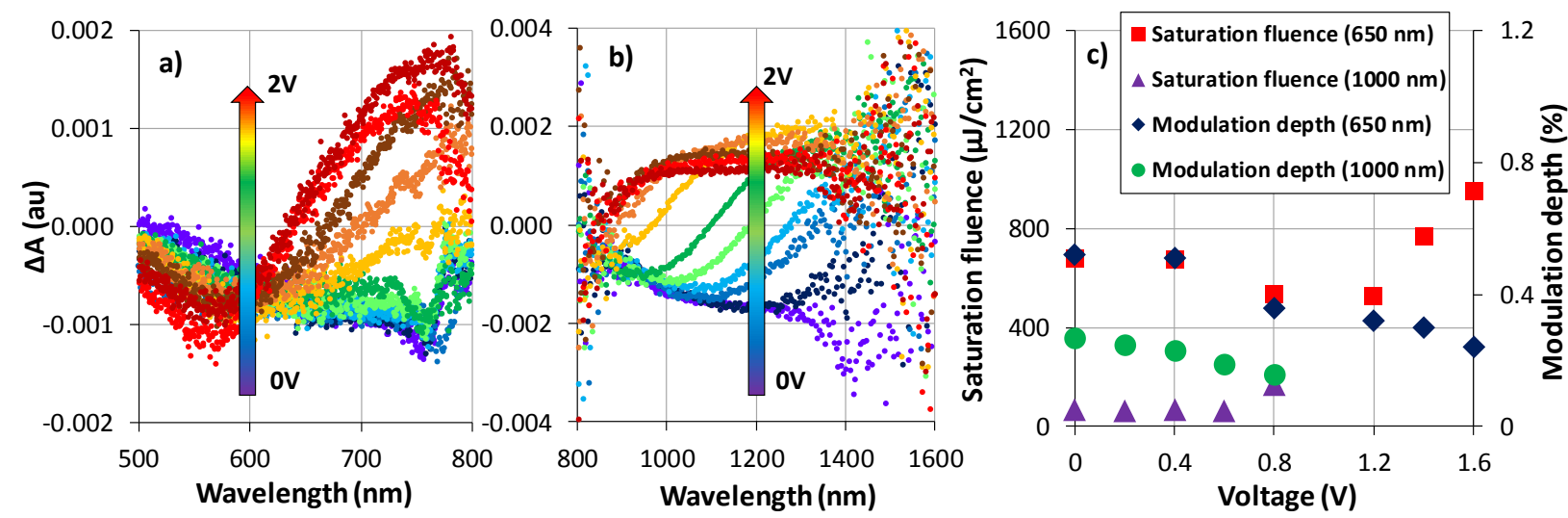

Fig.1. (a) Measured voltage-dependent nonlinear absorption $\triangle \mathrm{A}$ of the VCG-gold-SA for the visible and (b) near-infrared continuum probes. (c) Voltage-dependent modulation depth and saturation fluence as a function of applied bias at 650 and $1000 \mathrm{~nm}$.

The voltage-dependent ultrafast relaxation dynamics of the VCG-gold-SA were further investigated by using 470-nm and 750-nm pump pulses. Figure 2 summarizes the results obtained for the visible $(750 \mathrm{~nm})$ and nearinfrared $(1100 \mathrm{~nm})$ probes. As can be seen, we obtained the typical slow decay times on the order of 1-3 ps [5] for the bias voltages at which the interband transitions were still possible for the selected probe wavelengths. However, we observed an increase in the duration of the slow decay time from $0.9 \mathrm{ps}(0-1.2 \mathrm{~V})$ to around $10.7 \mathrm{ps}(1.4-2 \mathrm{~V})$, which possibly resulted from the multiphoton absorption at the visible probe wavelengths [see Fig. 2(a)]. For the near-infrared pump-probe measurements, the measured slow decay times of the VCG-gold-SA remained around $2.8 \pm 0.5 \mathrm{ps}$ as the applied bias was increased from 0 to $2 \mathrm{~V}$ [see Fig. 2(b)].
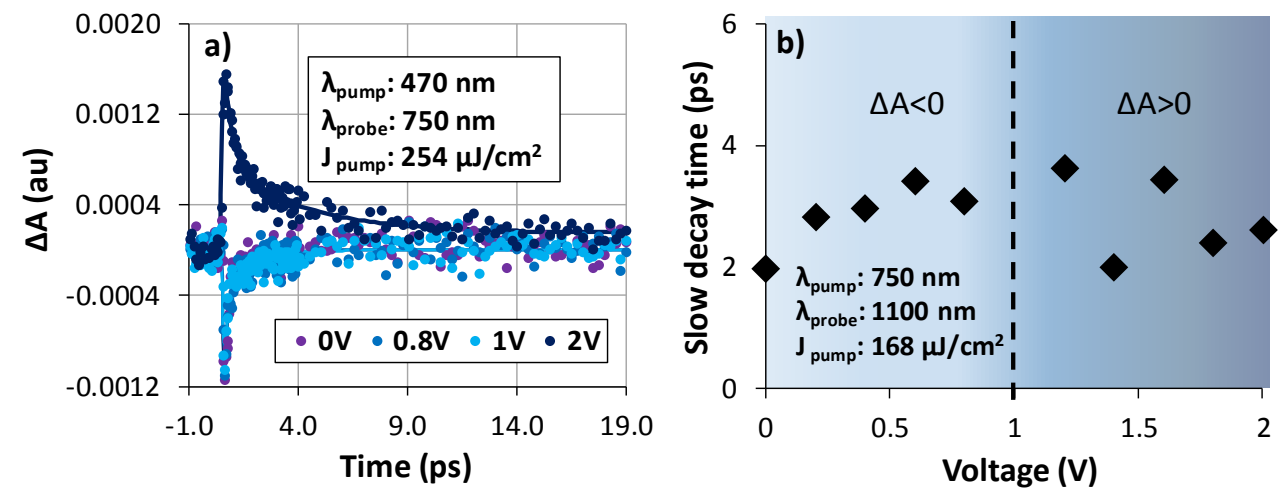

Fig.2. (a) Measured ultrafast relaxation dynamics of the VCG-gold-SA at the selected voltages for the visible probe. (b) Measured decay times as a function of the applied bias for the near-infrared probe.

In conclusion, by using femtosecond pump-probe spectroscopy, we have measured the saturable absorption bandwidth, saturation fluence, and modulation depth of a voltage-reconfigurable graphene-based supercapacitor structure at different bias voltages. Results indicate that at bias voltages where the device shows adjustable linear insertion loss, the saturable absorption still extends over an ultrabroad band. For example, at the bias voltage of $1 \mathrm{~V}$, the saturable absorption band was found to extend from $630 \mathrm{~nm}$ to $1100 \mathrm{~nm}$. These results indicate that VCG-goldSA functions effectively as a saturable absorber for femtosecond pulse generation over an ultrabroad wavelength range, even under applied bias voltages ( $0-2 \mathrm{~V}$ range) which are needed to adjust its linear insertion loss.

\section{References}

[1] A. Anton, J. Henri, D. Yunyun, W. Yadong, L. Harri, and S. Zhipei, "Nonlinear Optics with 2D Layered Materials," Adv. Mater., 1705963 (2018).

[2] I. Baylam, S. Ozharar, N. Kakenov, C. Kocabas, and A. Sennaroglu, "Femtosecond pulse generation from a Ti ${ }^{3+}:$ sapphire laser near $800 \mathrm{~nm}$ with voltage reconfigurable graphene saturable absorbers," Opt. Lett. 42, 1404 (2017).

[3] I. Baylam, O. Balci, N. Kakenov, C. Kocabas, and A. Sennaroglu, "Graphene-gold supercapacitor as a voltage controlled saturable absorber for femtosecond pulse generation," Opt. Lett. 41(2016).

[4] A. Marini, J. D. Cox, and F. J. Garcia de Abajo, "Theory of graphene saturable absorption," Phys. Rev. B 95, 125408 (2017).

[5] J. M. Dawlaty, S. Shivaraman, M. Chandrashekhar, F. Rana, and M. G. Spencer, "Measurement of ultrafast carrier dynamics in epitaxial graphene," Appl. Phys. Lett. 92, 042116 (2008). 